\title{
Ueber die Einwirkung des Chlors auf die
} Isonitrosoketone;

\author{
von L. Claisen und O. Manasse.
}

(Mittheilung ans dem chemischen Laboratorium der königl, Akademie der Wissensehaften zu München.)

Wie in früheren Abhandlungen ${ }^{1}$ ) gezeigt wurde, kann die Unwandlung der Ketone in Isonitrosoketone auf zweierlei Weise bewirkt werden: entweder durch Behandlung mit Amylnitrit und Natriumäthylat, wobei die Natriumsalze der Isonitrosoketone gebildet werden, oder durch Einwirkung von Amylnitrit bei Gegenwart von etwas Salzsäure. Von der Natur des Ketons hängt es $a b$, welches Verfahren vorzuziehen ist; für das Acetophenon, das Mesityloxyd und den Campher hat sich die erstere Art der Nitrosirung als die vortheilhaftere erwiesen; die Isonitrosoderivate des Acetons, des Diäthylketons und des Benzalacetons dagegen konnten in befriedigender Ausbeute nur nach dem zweiten Verfahren gewonnen werden. Bei letzterem genügt, wie dies schon früher ${ }^{2}$ ) mitgetheilt wurde, eine kleine Menge Salzsäure, um dio Reaction einzuleiten und bis zum Ende durchzuführen; die Umsetzung verläuft offenbar so, dass zunächst Amylalkohol und Nitrosylchlorid gebildet wird, welch' letzteres dann unter Regenerirung von Salzsäure die Nitrosirung des Ketons bewirkt:

$$
\mathrm{R}-\mathrm{CO}-\mathrm{CH}_{3}+\mathrm{NOCl}=\mathrm{R}-\mathrm{CO}-\mathrm{CH}=\mathrm{NOH}+\mathrm{HCl} .
$$

Durch die zurückgebildete Salzsäure wird eine neue Menge von Amylnitrit in Nitrosylchlorid verwandelt und so fort, bis alles Nitrit verbraucht ist.

Ueberschüssige Salzsäure anzuwenden ist nicht nur zwecklos, sondern sogar schädlich, indem dieselbe durch die salpetrige

) L. Claisen, Ber. d. deutsch. chem. Ges. 20, 252 u. 656; I. Claisen u. O. Manasse, Ber. d. deutsch, chem. Ges. 20. $2194 ; 22,526$ u. 530.

2) Ber. d. deutsch. chem. Ges. 22, 527. 
Säure zu Chlor oxydirt wird und so die Bildung von gechlorten Isonitrosolietonen veranlasst. Einen solchen abweichenden Verlauf der Reaction haben wir beim Acetophenon beobachtet, als wir eine Mischung desselben mit Amylnitrit (in äquimolekularem Verhältniss) mit der gleichen Gewichtsmenge von rauchender Salzsäure einige Zeit unter Eiskübiung stehen liessen. Die Mischung färbte sich braunroth und es fand ein langsames Aufsteigen von Gasbläschen statt. Nach halbstündiger Kinwirkung wurde die Flüssigkeit mit niedrigsiedendem Ligroïn versetzt, gut damit durchgeschüttelt und noch weitere 12 Stunden in Eiswasser stehen gelassen. Alsdann hatte sich (in allerdings nur mässiger Menge) eine krystallinische Verbindung abgeschieden, welche abgesaugt und aus wenig heissem Benzol umkrystallisirt wurde. Die Analyse der so gereinigten und dann bei $131-132^{\circ}$ schmelzenden Substanz ergab Zahlen, welche der Zusammensetzung eines Monochlor-Isonitrosoacetophenons $\mathrm{C}_{8} \mathrm{H}_{6} \mathrm{ClO}_{2} \mathrm{~N}$ entsprechen:

I. $0,2251 \mathrm{~g}$ gaben $0,4278 \mathrm{CO}_{2}$ und $0,0744 \mathrm{H}_{2} \mathrm{O}$.

II. $0,117 \mathrm{~g} \quad, \quad 0,0915 \mathrm{AgCl}$.

III. $0,1314 \mathrm{~g} \quad, \quad 0,1027 \mathrm{AgCl}$.

\begin{tabular}{cccc} 
& $\begin{array}{c}\text { Berechnet für } \\
\mathrm{C}_{8} \mathrm{H}_{6} \mathrm{ClO}, \mathrm{N}\end{array}$ & \multicolumn{2}{c}{ Gefunden } \\
$\mathrm{C}$ & $\check{2} 2,32$ & $\mathrm{I}$. & $\mathrm{II}$. \\
$\mathrm{H}$ & 3,27 & 51,84 & - \\
$\mathrm{Cl}$ & 19,34 & 3,67 & - \\
$\mathrm{N}$ & $\tau, 63$ & 19,34 & 19,33
\end{tabular}

Die Verbindung ist schwer löslich in Ligroïn, ziemlich leicht löslich dagegen in heissem Benzol, aus welchem sie sich beim Erkalten in perlmutterglänzenden Blättchen abscheidet. Im Gegensatz zum Isonitrosoacetophenon wird sie von Natronlauge erst in der Wärme gelöst und zwar unter Zersetzung und Bildung von Natriumbenzoat. Bei andauerndem Schütteln mit wässrigem kohlensaurem Natron zergeht sie zu öligen Tropfen, die auch durch längeres Erwärmen nur schwierig in Lösung $\mathrm{zu}$ bringen sind. Amylnitrit, welches Isonitroso- 
acetophenon so leicht zersetzt, wirkt selbst in der Wärme kaum auf dieses Chlorderivat ein; durch Zufügen von Ligroün kann man es aus der Mischung wieder unverändert ausfällen.

Viel bequemer und reichlicher als nach dem angegebenen Verfahren kann die Substanz durch Chloriren von fertig gebildetem Isonitrosoacetophenon erhalten werden, was insofern wichtig ist, als daraus mit Sicherheit hervorgeht, dass der Körper in der That ein Monochlorsubstitutionsproduct des Isonitrosoacetophenons und nicht etwa ein Salzsäureadditionsproduct $\mathrm{C}_{8} \mathrm{H}_{8} \mathrm{ClO}_{2} \mathrm{~N}$ darstellt, das sich ja unter den geschilderten Verhältnissen leicht hätte bilden können. Beide Körper weichen nämlich in der Zusammensetzung so wenig von einander $a b$, dass die Analysen allein keine sichere Entscheidung bezüglich der Formel gestatten.

$\begin{array}{lcc} & \text { Berechnot für } & \text { Berechnet für } \\ & \mathrm{C}_{8} \mathrm{H}_{8} \mathrm{O}_{9} \mathrm{NCl} & \mathrm{C}_{8} \mathrm{~B}_{8} \mathrm{O}_{2} \mathrm{NCl} \\ \mathrm{C} & 532,32 & 5 \mathrm{~s}, 75 \\ \mathrm{H} & 3,27 & 4,31 \\ \mathrm{Cl} & 19,34 & 19,14 \\ \mathrm{~N} & 7,63 & 7,55\end{array}$

Zur Darstellung des Chlorproductes auf diesem letzteren Wege wird in eine Chloroformlösung des Isonitrosoacetophenons so lange Chlorgas eingeleitet, bis die Abscheidnng von Krystallen beginnt, welche man durch Zufügen von Ligroïn vervollständigt. Durch Umkrystallisiren aus heissem Benzol wurde auch hier die Verbindung in perlmutterglänzenden Blättchen vom Schmelzpunkt $132^{\circ}$ erhalten. Die Ausbeute betrug circa $70 \mathrm{pC}$. vom Gewicht des angewandten Isonitrosoketons.

Bezüglich der Constitution des Körpers hatte man die Wahl zwischen den Formeln:
1) $\mathrm{C}_{6} \mathrm{H}_{4} \mathrm{Cl}-\mathrm{CO}-\mathrm{CH}=\mathrm{NOH}$
2) $\mathrm{C}_{6} \mathrm{H}_{5}-\mathrm{CO}-\mathrm{CCl}=\mathrm{NOH}$

3) $\mathrm{C}_{6} \mathrm{H}_{5}-\mathrm{CO} \cdot \mathrm{CH}=\mathrm{N}-\mathrm{O}-\mathrm{Cl}$.

Dic erste derselben kommt in Wegfall, weil die Verbindung bei der Zersetzung mit Alkalien nicht ChlorbenzoëAnnalen der Chemie 274. Bd. 
săure, sondern Benzoësänre liefert. Zwischen den beiden anderen Formeln konnte leicht durch die folgende Beobachtung entschieden werden. Es zeigte sich, dass Isonitrosoaceton sich gegen Chlor ganz ebenso verhält wie das Acetophenonderivat. Als wir in eine Lösung von Isonitrosoaceton in der fünffachen Gewichtsmenge Chloroform solange Chlorgas einleiteten, bis die Gewichtszunahme der Bildung eines Monochlorderivats entsprach, schied sich nach einigem Stehen eine krystallinischkörnige Masse ab, welche abgesangt, mit Ligroin gewaschen und aus Benzol umkrystallisirt wurde. So gereinigt, schmolz die Substanz bei $109^{\circ}$ und erwies sich als völlig identisch mit einer Verbindung, welche zuerst von Glutz ${ }^{3}$ ) und Barbagli a ${ }^{4}$ ) durch Einwirkung von Salpetersäure auf Chloraceton dargestellt worden ist; später wurde sie von Sandmeyer ${ }^{5}$ ) durch successive Behandlung des Acetons mit salpetriger Süure und Salzsäure erhalten. Nach der ersteren Bildungsweise (aus Chloraceton) kann der Körper wohl nur als ein gechlortes Isonitrosoaceton von folgender Formel $\mathrm{CH}_{3}-\mathrm{CO}-\mathrm{CCl}=\mathrm{NOH}$ betrachtet werden, womit auch die von Sandmeyer bewirkte Ueberführung in Brenztraubensäure in gutem Einklange steht. Da nun, wie gezeigt wurde, das Chlornitrosoacetophenon unter genau denselben Bedingungen erhalten werden kann wie das Chlornitrosoaceton, wird man seine Constitution auch durch die entsprechende Formel

ausdrücken müssen.

$$
\mathrm{C}_{0} \mathrm{H}_{-}-\mathrm{CO}-\mathrm{CCl}=\mathrm{NOH}
$$

3) Zeitschrift für Chemie 1870, כ29.

^) Ber. d. deutsch. chem. Ges. 6, 321 .

5) Ber. d. deutsch. chom. Ges. 20, 689. 